\title{
The Earth, Planets and Space Special Issue: "Science of solar system materials examined from Hayabusa and future missions"
}

\author{
Tatsuaki Okada ${ }^{*}$, Michael E. Zolensky ${ }^{2}$, Trevor R. Ireland ${ }^{3}$ and Toru Yada ${ }^{1}$
}

It is our great pleasure to present the special issue of the journal Earth, Planets and Space, entitled "Science of solar system materials examined from Hayabusa and future missions." We would like to start with a brief introduction of the Hayabusa mission and its story of sample collection on the asteroid, followed by the curation and delivery of returned samples to researchers. All of the 14 manuscripts published in this special issue are also reviewed here.

Hayabusa was the Japan Aerospace Exploration Agency (JAXA) engineering mission to explore and return a sample from a near-Earth asteroid. It was launched in 2003 by 5th $\mathrm{M}-\mathrm{V}$ launch vehicle, visited and explored asteroid 25143 Itokawa in 2005, and finally returned surface materials from there to Earth in 2010. The purpose of this mission was an engineering experiment of a round-trip journey to the asteroid, as well as scientific experiments for understanding the origin and evolution of the early solar system by exploring a primitive body. The S-type, sub-kilometer-sized asteroid Itokawa was found to be chondritic in composition and low-density rubble-pile in structure (e.g., Fujiwara et al. 2006). Its surface was basically rough and covered with boulders, but smooth terrains such as MUSES-C Regio also existed where the first and second touchdown operations were conducted for sample collection (see Fig. 1).

The returned samples have been curated in JAXA's Extraterrestrial Sample Curation Center. These samples are of small amount and very fine grained, because the impact-sampling method failed to shoot projectiles for sample acquisition and only floating dust grains entered into the sample catcher (see Fig. 2). Thousands of micron-sized silicate-dominated grains other than artificial contaminants were found by scraping them from the

\footnotetext{
* Correspondence: okada@planeta.sci.isas.jaxa.jp

'Institute of Space and Astronautical Science, Japan Aerospace Exploration Agency, Sagamihara 252-5210, Japan

Full list of author information is available at the end of the article
}

inside wall of the catcher with a Teflon spatula and tapping on the chamber. These samples are consistent with chondritic composition as shown by scanning electron microscopy (SEM) with energy-dispersive X-ray spectroscopy (EDS). The removal of tens of micron-sized particles (up to $300 \mu \mathrm{m}$ ) in the catcher was started using the newly developed electrostatic control manipulator. So far, about 500 particles have been catalogued for initial description about size, shape, SEM images, and representative elemental and mineral composition from EDS spectra (e.g., Yada et al. 2014), and part of those catalogued particles have been delivered for more advanced research including preliminary examinations and through an international announcement of opportunity.

The preliminary examination of Hayabusa-returned samples has successfully unveiled the mineralogical, petrographic, chemical, and isotopic relationships between an S(IV)-type asteroid and ordinary LL chondrite meteorites as predicted by ground-based observation (e.g., Binzel et al. 2001, 2010) and provided the first direct evidence that meteorites originate from asteroids (Ebihara et al. 2011; Nakamura et al. 2011; Noguchi et al. 2011; Tsuchiyama et al. 2011; Yurimoto et al. 2011). The particle-size distribution and existence of rounded grains (Tsuchiyama et al. 2011) as well as the noble gas isotopic compositions (Nagao et al. 2011) have also recorded asteroid surface processes such as meteoroid impacts, possible granular flow, and solar wind irradiation on the asteroid surface, which were not observed in meteorites. Organic analyses have been also performed (Kitajima et al. 2011; Naraoka et al. 2012), but indigenous organic compounds have not been identified from the samples to date.

Investigation of Hayabusa-returned samples has expanded to any interested researchers by applying to the International Announcement of Opportunity, and the successful winners started their advanced and sophisticated research. Hayabusa 2013: 1st Symposium of Solar 




Fig. 1 Digital image of asteroid 25143 Itokawa and the estimated first (high-latitude region) and second (equatorial region) touchdown sites. Both sites are inside a flat terrain MUSES-C Regio. The image was taken from Home Position with ONC-T imager on 10 October 2005. (ST_2448357351_v, http://darts.isas.jaxa.jp/planet/project/hayabusa/ amica.pl)

System Materials was held for these researchers to come together and discuss their latest results, giving new prospects to understanding the asteroid-meteorite connection and the early solar system origin and evolution. All the papers presented in the symposium were invited for submission to this EPS special issue, and any papers related to the topics were also welcomed. In the end, 14 manuscripts were successfully completed for publication in this special issue.

Among the important topics discussed here are the surface processes discovered in the ultrafine structures of Hayabusa-returned samples, influenced by space weathering or shock or thermal alteration mechanism. This was enabled by advanced analytical techniques for ultrafine grains using transmission electron microscopy

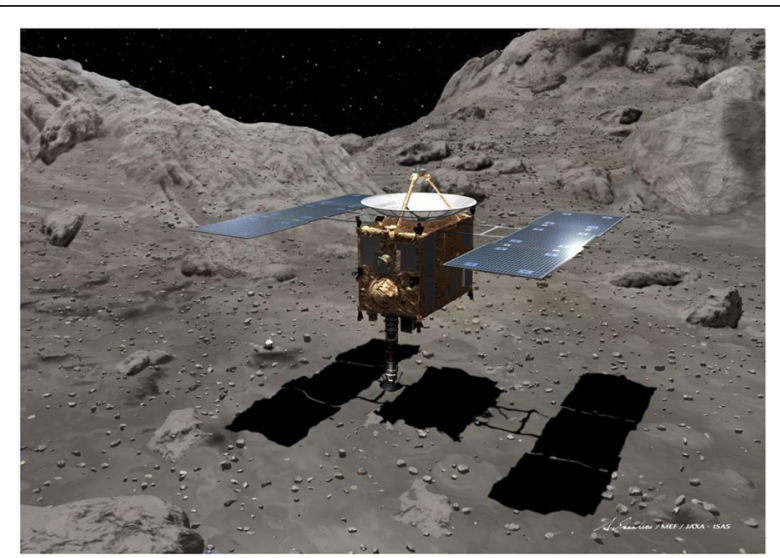

Fig. 2 An artist view of the Hayabusa spacecraft just a moment before the touchdown on the surface of asteroid 25143 Itokawa for sampling with the horn-shaped sampler (Ikeshita/MEF/JAXA-ISAS). Actual result was that the impact sampling failed to shoot the projectile for excavating the surface materials and collecting part of them into the sample catcher, but only the dusts floating around there were obtained and consequently returned to Earth after preparing ultrathin-sectioned specimens using ultramicrotomy and focused ion beam techniques.

Keller and Berger (2014) report on their detailed structural and elemental analysis by scanning and transmission electron microscopy of two particles returned by Hayabusa, which have a 50- to 100 -nm-thick disordered olivine rim surrounding the particles. This evidence of space weathering by irradiation of solar wind ions and the number density of ion particle track in the rim suggest the short exposure age of $10^{3}$ to $10^{4}$ years. Noguchi et al. (2014) report results of detailed mineralogical investigation of four Itokawa particles collected from the first touchdown site by micro-Raman spectroscopy, scanning electron microscopy, electron microprobe analysis, $\mathrm{X}$-ray absorption spectroscopy, and transmission electron microscopy and conclude that those particles are consistent with LL6 and that space weathering (space-weathered rim and number densities of solar flare tracks) at this site was less severe than at the second touchdown site reported in the initial analysis (Noguchi et al. 2011), which suggests uncertainty in the previously reported exposure history of the surface, and $10^{3}$ years rather than $10^{4}$ years of exposure age. Thompson et al. (2014) report detailed microchemical and microstructural features indicative of space weathering in a Hayabusa-returned particle using transmission electron microscopy of the microtomed ultrathin section of rim regions. They suggest that solar wind irradiation is likely responsible for the surface amorphous regions and the multi-layered rims with a nano-crystalline outer layer underlain by an amorphous inner layer are derived both from micrometeorite impacts and solar wind irradiation, indicating multiple surface-processing events on the asteroid. Langenhorst et al. (2014) report detailed analytical scanning and transmission electron microscopic investigations on one olivine-dominated Itokawa particle, which suggests that the particle is of LL-chondrite origin as previously reported and that even regolith particles lacking visible microcraters on their surfaces might have still experienced shock metamorphism and were involved in collisional fragmentation. In Harries and Langenhorst (2014), analysis of another Itokawa particle suggests a lower abundance of shock metamorphism relative to the one by Langenhorst et al. (2014), indicating heterogeneity of shock events on Itokawa or on its parent body. A thin polycrystalline olivine rim on one face of the particle may have originated by solar wind irradiation, followed by annealing and recrystallization, which may have occurred due to the warmer surface temperature of Itokawa near perihelion distances by dynamical orbital evolution.

New analyses and discussion also strengthen the previous results and contribute to building the new prospects of asteroid and solar system science. Mikouchi et al. (2014) report the results of a mineralogical investigation 
of seven Itokawa particles collected from the first touchdown site of Itokawa through field emission secondary electron microscopy, synchrotron radiation X-ray diffraction, and $\mathrm{X}$-ray absorption near-edge structure analysis and concluded that the particles are slightly shocked equilibrated LL chondrite, as suggested in the preliminary examination. Connolly et al. (2015) discussed the formation of rounded shape grains included in the samples returned by Hayabusa (e.g., Tsuchiyama et al. 2011) and argued that the Itokawa surface was in a high-energy environment possibly caused by the YORP effect to raise a continuous granular flow. In their hypothesis, the portion of rounded to angular grains is higher for C-type relative to S-type asteroids, which could be verified by samples returned by Hayabusa 2 or OSIRIS-REx. Takeda et al. (2015) report on the mineralogy of three LL chondrites (possibly related to Itokawa) that partial melts found in them indicate that even a primitive solar system parent body of such as LL chondrites experienced partial melting and suggests that granulitic materials may have been formed at depths within their parent body by an impact event.

Discussions concerning the category 3 particles are specially featured topics in this special issue. Hayabusareturned samples are classified into four categories. Category 1 and 2 particles consist of silicate minerals without or with a metallic component, respectively, and most of them are considered to have been indigenous to Itokawa. Category 4 includes artificial materials from inside the sample catcher or from the sampling tools. Category 3 particles from Itokawa represent a number of samples that are predominantly composed of carbon or carbon compounds. The lack of carbon in any of the type I or II grains that have been proven to originate on Itokawa has raised suspicion that the category 3 particles are contaminants of possibly terrestrial origin or are materials that may have originated from the surface of the Hayabusa spacecraft. In a series of five studies in this issue, the possible origins of category 3 particles are addressed.

Uesugi et al. (2014) report preliminary results of the analyses of five category 3 (carbonaceous) materials removed from the Hayabusa spacecraft sample catcher and conclude that differences in their microstructure and elemental distributions suggest multiple origins. They also inform the summary of damage occurring on those samples by each analytical process as lessons learned for future sample return analysis of carbonaceous materials expected by the Hayabusa 2 and OSIRIS-REx missions. Ito et al. (2014) have measured H, C, and N isotopic compositions of three category 3 particles with ion imaging on a nanoSIMS ion microprobe. Isotopic compositions of these elements are quite variable in extraterrestrial samples; however, they have found that the isotopic compositions are all within error of terrestrial.
Kitajima et al. (2015) performed micro-Raman and infrared analysis of the same three particles as measured by Ito et al. (2014). They found that the Raman spectra show disordered parameters associated with a low-maturity level, and hence, the particles are not associated with the Itokawa asteroid particles. Naraoka et al. (2015) report TOF-SIMS analysis of three category 3 particles [only one in common with Ito et al. (2014) and Kitajima et al. (2015)] in order to determine elemental compositions. These analyses have proven to be difficult because the particles have been part of an extended analytical program, and in particular, the Cs primary ion beam used in the nanoSIMS analysis has pervaded the grain. Nevertheless, Naraoka et al. (2015) found organic carbon associated with nitrogen, fluorine, and silicon, which are uncommon in nature but quite common in man-made materials. They concluded that the category 3 particles are likely manmade rather than natural organic matter. Based on these studies, it appears that the category 3 particles are not associated with Itokawa but their specific site of origin is difficult to determine. On the other hand, Yabuta et al. (2014) report results of analyses by scanning transmission X-ray microscopy using carbon-, nitrogen-, oxygen-, fluorine-, and calcium-X-ray absorption near-edge structure (XANES) spectroscopy for different kinds of two carbonaceous particles removed from the Hayabusa spacecraft sample catcher. These were distinct from commercial or biologic fresh materials, but they were unable to rule out an extraterrestrial origin for some of these particles.

Additional featured topics discussed in the symposium and also in this special issue are instrumentation and methods for sample analysis, as well as remote sensing or ground-based observations for asteroids, comets, and meteors. The only such manuscript published in this special issue is by Mediedo (2014), which reports the newly developed ground robotic spectroscopic observation system for determining the composition of fireballs, the solar system materials ablating during atmosphere entry. The derived physico-chemical data are expected to contribute to a systematic characterization of materials coming from different comets and asteroids.

All the 14 manuscripts in this special issue are fruitful and constructive for discussion about the asteroid-meteorite connection and the early solar system origin and evolution. Detailed analysis of Hayabusa-returned samples is not yet complete and will continue with more sophisticated and more statistically significant analyses and techniques. In the near future, sample returns from C-type asteroid 1999JU3 by Hayabusa 2 in 2020 and from B-type asteroid Bennu by OSIRIS-Rex in 2023 are expected to advance asteroid and solar system science, and we believe that the developed methodology and lessons learned from the analysis of the Hayabusa-returned samples will play a significant role in these missions. 


\section{Author details}

${ }^{1}$ Institute of Space and Astronautical Science, Japan Aerospace Exploration Agency, Sagamihara 252-5210, Japan. ${ }^{2}$ Astromaterials Research and Exploration Science, NASA Johnson Space Center, Houston, TX 77058, USA. ${ }^{3}$ Research School of Earth Sciences, The Australian National University, Canberra ACT 0200, Australia.

\section{Received: 19 April 2015 Accepted: 19 April 2015}

Published online: 26 July 2015

\section{References}

Binzel RP, Rivkin AS, Bus SJ, Sunshine JM, Burbine TH (2001) MUSES-C target asteroid (25143) 1998 SF36: a reddened ordinary chondrite. Meteorit Planet Sci 36:1167-1172

Binzel RP, Morbidelli A, Merouane S, DeMeo FE, Birlan M, Vernazza P, Thomas CA, Rivkin AS, Bus SJ, Tokunaga AT (2010) Earth encounters as the origin of fresh surfaces on near-Earth asteroids. Nature 463:331-334

Connolly HC Jr, Lauretta DS, Walsh KJ, Tachibana S, Bottke WF Jr (2015) Towards understanding the dynamical evolution of asteroid 25143 Itokawa: constraints from sample analysis. Earth Planets Space 67:12. doi:10.1186/s40623-015-0185-3

Ebihara M, Sekimoto S, Shirai N, Hamajima Y, Yamamoto M, Kumagai K, Oura Y, Ireland TR, Kitajima F, Nagao K, Nakamura T, Naraoka H, Noguchi T, Okazaki R, Tsuchiyama A, Uesugi M, Yurimoto H, Zolensky ME, Abe M, Fujimura A, Mukai T, Yada T (2011) Neutron activation analysis of a particle returned from asteroid Itokawa. Science 333:1119-1121

Fujiwara A, Kawaguchi J, Yeomans DK, Abe M, Mukai T, Okada T, Saito J, Yano H, Yoshikawa M, Scheeres DJ, Barnouin-Jha O, Cheng AF, Demura H, Gaskell RW, Hirata N, Ikeda H, Kominato T, Miyamoto H, Nakamura AM, Nakamura R, Sasaki S, Uesugi K (2006) The rubble-pile asteroid Itokawa as observed by Hayabusa. Science 312:1330-1334

Harries D, Langenhorst F (2014) The mineralogy and space weathering of a regolith grain from 25143 Itokawa and the possibility of annealed solar wind damage. Earth Planets Space 66:163

Ito M, Uesugi M, Naraoka H, Yabuta H, Kitajima F, Mita H, Takano Y, Karouji Y, Yada T, Ishibashi Y, Okada T, Abe M (2014) H, C, and N isotopic compositions of Hayabusa category 3 organic samples. Earth Planets Space 66:91

Keller L, Berger E (2014) A transmission electron microscope study of Itokawa regolith grains. Earth Planets Space 66:71

Kitajima F, Kotsugi M, Ohkochi T, Naraoka H, Ishibashi Y, Abe M, Fujimura A, Okazaki R, Yada T, Nakamura T, Noguchi T, Nagao K, Tsuchiyama A, Mukai T, Sandford SA, Okada T, Shirai K, Ueno M, Yoshikawa M, Kawaguchi J (2011) A micro-spectroscopic approach to the carbonaceous matter in the particles recovered by the Hayabusa mission, (Abstract \#1855), 42nd Lunar and Planetary Science Conference

Kitajima F, Kotsugi M, Ohkochi T, Naraoka H, Ishibashi Y, Uesugi M, Karouji Y, Abe M, Fujimura A, Yada T, Okazaki R, Nakamura T, Noguchi T, Nagao K, Tsuchiyama A Yurimoto H, Ebihara M, Ito M, Yabuta H, Mita H, Takano Y, Mukai T, Sandford SA, Okada T, Shirai K, Ueno M, Yoshikawa M, Kawaguchi J (2015) A micro-Raman and infrared spectroscopic approach to the several stony and organic (category 3) particles recovered by the Hayabusa mission. Earth Planets space 67:20

Langenhorst F, Harries D, Pollok P, van Aken A (2014) Mineralogy and defect microstructure of an olivine-dominated Itokawa dust particle: evidence for shock metamorphism, collisional fragmentation, and LL chondrite origin. Earth Planets Space 66:118

Mediedo JM (2014) Robotic systems for the determination of the composition of solar system materials by means of fireball spectroscopy. Earth Planets Space $66: 70$

Mikouchi T, Komatsu M, Hagiya K, Ohsumi K, Zolensky ME, Hoffmann V, Martinez J, Hochleitner R, Kaliwoda M, Terada Y, Yagi N, Takata M, Satake W, Aoyagi Y, Takenouchi A, Karouji Y, Uesugi M, Yada T (2014) Mineralogy and crystallography of some Itokawa particles returned by the Hayabusa asteroidal sample return mission. Earth Planets Space 66:82

Nagao K, Okazaki R, Nakamura T, Miura YN, Osawa T, Bajo K, Matsuda S, Ebihara M, Ireland TR, Kitajima F, Naraoka H, Noguchi T, Tsuchiyama A, Yurimoto H, Zolensky ME, Uesugi M, Shirai K, Abe M, Yada T, Ishibashi Y, Fujimura A, Mukai T, Ueno M, Okada T, Yoshikawa M, Kawaguchi J (2011) Irradiation history of Itokawa regolith material deduced from noble gases in the Hayabusa samples. Science 333:1128-1131

Nakamura T, Noguchi T, Tanaka M, Zolensky ME, Kimura M, Tsuchiyama A, Nakato A, Ogami T, Ishida H, Uesugi M, Yada T, Shirai S, Fujimura A, Okazaki R, Sandford
SA, Ishibashi Y, Abe M, Okada T, Ueno M, Mukai T, Yoshikawa M, Kawaguchi J (2011) Itokawa dust particles: a direct link between S-type asteroids and ordinary chondrites. Science 333:1113-1116

Naraoka H, Mita H, Hamase K, Mita M, Yabuta H, Saito K, Fukushima K, Kitajima F, Sandford SA, Nakamura T, Noguchi T, Okazaki R, Nagao K, Ebihara M, Yurimoto H, Tsuchiyama A, Abe M, Shirai K, Ueno M, Yada T, Ishibashi Y, Okada T, Fujimura A, Mukai T, Yoshikawa M, Kawaguchi J (2012) Preliminary organic compound analysis of microparticles returned from asteroid 25143 Itokawa by the Hayabusa mission. Geochem J 46:61-72

Naraoka H, Aoki D, Fukushima K, Uesugi M, Ito M, Kitajima F, Mita H, Yabuta H, Takano Y, Yada T, Ishibashi Y, Okada T, Abe M (2015) ToF-SIMS analysis of carbonaceous particles in the sample capsule of the Hayabusa mission, Earth Planet Space in press

Noguchi T, Nakamura T, Kimura M, Zolensky ME, Tanaka M, Hashimoto T, Konno M, Nakato A, Ogami T, Fujimura A, Abe M, Yada T, Mukai T, Ueno M, Okada T, Shirai K, Ishibashi Y, Okazaki R (2011) Incipient space weathering observed on the surface of Itokawa dust particles. Science 333:1121-1125

Noguchi T, Bridges JC, Hicks LJ, Gurman SJ, Kimura M, Hashimoto T, Konno M, Bradley JP, OkazakiR UM, Yada T, Karouji Y, Abe M, Okada T, Mitsunari T, Nakamura T, Kagi H (2014) Mineralogy of four Itokawa particles collected from the first touchdown site. Earth Planets Space 66:124

Takeda H, Nagaoka H, Yamaguchi A, Karouji Y, Yazawa Y (2015) Mineralogy of some evolved LL chondrites with reference to asteroid materials and solar system evolution. Earth Planets Space 67:5

Thompson MS, Christoffersen R, Zega TJ, Keller LP (2014) Microchemical and structural evidence for space weathering in soils from asteroid Itokawa. Earth Planets Space 66:89

Tsuchiyama A, Uesugi M, Matsushima T, Michikami T, Kadono T, Nakamura T, Uesugi K, Nakano T, Sandford SA, Noguchi R, Matsumoto T, Matsuno J, Nagano T, Imai Y, Takeuchi A, Suzuki Y, Ogami T, Katagiri J, Ebihara M, Ireland TR, Kitajima F, Nagao K, Naraoka H, Noguchi T, Okazaki R, Yurimoto H, Zolensky ME, Mukai T, Abe M, Yada T et al (2011) Three-dimensional structure of Hayabusa samples: origin and evolution of Itokawa regolith. Science 333:1121-1125

Uesugi M, Naraoka M, Ito M, Yabuta H, Kitajima F, Takano Y, Mita H, Ohnishi I, Kebukawa Y, Yada T, Karouji Y, Ishibashi Y, Okada T, Abe M (2014) Sequential analysis of carbonaceous materials in Hayabusa-returned samples for the determination of their origin. Earth Planets Space 66:102

Yabuta H, Uesugi M, Naraoka H, Ito M, Kilcoyne ALD, Sandford SA, Kitajima F, Mita H, Takano Y, Yada T, Karouji Y, Ishibashi Y, Okada T, Abe M (2014) X-ray absorption near edge structure spectroscopic study of Hayabusa category 3 carbonaceous particles. Earth Planets Space 66:156

Yada T, Fujimura A, Abe M, Nakamura T, Noguchi T, Okazaki R, Nagao K, Ishibashi Y, Shirai K, Zolensky ME, Sandford S, Okada T, Uesugi M, Karouji Y, Ogawa M, Yakame S, Ueno M, Mukai T, Yoshikawa M, Kawaguchi J (2014) Hayabusa returned sample curation in the planetary material sample curation facility of JAXA. Meteorit Planet Sci 49:135-153

Yurimoto H, Abe K, Abe M, Ebihara M, Fujimura A, Hashiguchi M, Hashizume K, Ireland TR, Itoh S, Katayama J, Kato C, Kawaguchi J, Kawasaki N, Kitajima F, Kobayashi S, Meike T, Mukai T, Nagao K, Nakamura T, Naraoka H, Noguchi T, Okazaki R, Park C, Sakamoto N, Seto Y, Takei M, Tsuchiyama A, Uesugi M, Wakaki S, Yada T et al (2011) Oxygen isotopic compositions of asteroidal materials returned from Itokawa by the Hayabusa mission. Science 333:1116-1119

\section{Submit your manuscript to a SpringerOpen ${ }^{\circ}$ journal and benefit from:}

- Convenient online submission

Rigorous peer review

- Immediate publication on acceptance

- Open access: articles freely available online

- High visibility within the field

- Retaining the copyright to your article

Submit your next manuscript at $>$ springeropen.com 